\title{
In vitro and in silico Approaches to Study Cytochrome P450-Mediated Interactions
}

\author{
Boon Hooi Tan ${ }^{1}$, Yan Pan², Amelia Nathania Dong ${ }^{3}$ and Chin Eng Ong ${ }^{3}$ \\ ${ }^{1}$ School of Health Sciences, International Medical University, Jalan Jalil Perkasa, Bukit Jalil, Kuala Lumpur, Malaysia; ${ }^{2}$ \\ Department of Biomedical Science, University of Nottingham Malaysia Campus, Jalan Broga, Semenyih, Selangor, \\ Malaysia; ${ }^{3}$ School of Pharmacy, Monash University Malaysia, Jalan Lagoon Selatan, Bandar Sunway, Selangor, Malaysia
}

Received, May 8, 2017; Revised, July 31, 2017; Accepted, August 25, 2017; Published August 27, 2017.

\begin{abstract}
In vitro and in silico models of drug metabolism are utilized regularly in the drug research and development as tools for assessing pharmacokinetic variability and drug-drug interaction risk. The use of in vitro and in silico predictive approaches offers advantages including guiding rational design of clinical drug-drug interaction studies, minimization of human risk in the clinical trials, as well as cost and time savings due to lesser attrition during compound development process. This article gives a review of some of the current in vitro and in silico methods used to characterize cytochrome P450(CYP)-mediated drug metabolism for estimating pharmacokinetic variability and the magnitude of drug-drug interactions. Examples demonstrating the predictive applicability of specific in vitro and in silico approaches are described. Commonly encountered confounding factors and sources of bias and error in these approaches are presented. With the advent of technological advancement in high throughput screening and computer power, the in vitro and in silico methods are becoming more efficient and reliable and will continue to contribute to the process of drug discovery, development and ultimately safer and more effective pharmacotherapy.
\end{abstract}

This article is open to POST-PUBLICATION REVIEW. Registered readers (see "For Readers") may comment by clicking on ABSTRACT on the issue's contents page.

\section{INTRODUCTION}

Members of the cytochrome P450 (CYP) superfamily are membrane-bound, haem-containing terminal oxidases which are responsible for the metabolic activation or inactivation of drugs, toxins as well as endogenous compounds. Over the past two decades, numerous advances have been achieved on the use of in vitro approaches to investigate drug metabolism and drug interaction involving CYPs. These approaches have been shown effective in predicting the potential clinical drug-drug interactions (DDIs). With the construction of recombinant human CYPs using various expression systems [1], the source of enzyme is no longer limited to liver microsomes, liver slices or hepatocytes. Moreover, the activity of a single CYP can be studied specifically using recombinant CYPs without the requirement of highly specific probe substrates. The availability of these enzyme sources, coupled with the use of in vitro CYP assays and a variety of modern analytical techniques, have permitted delineation of enzyme activity, induction and inhibition. The in silico approaches have emerged, over the past decade or so, as tools of increasing importance to predict drug metabolism and interaction [2]. Different from in vitro approaches, computational approaches can be applied to hypothetical compounds as their three dimensional (3D) structures can readily be generated in silico. Similarly the availability of crystal structures of major human CYPs has allowed molecular modeling to decipher binding, docking and interaction conformation and affinity of different compounds (ligands) with the generated CYP structures. Therefore in silico methods help producing molecular data and filling information void in experimentally derived data, and represent an important component in examining CYP-mediated DDI risk and mechanism.

This article gives an overview of the current in vitro and in silico methods to investigate CYP-

Corresponding Author: Associate Professor Dr Chin Eng Ong; School of Pharmacy; Monash University Malaysia; Jalan Lagoon Selatan; 47500 Bandar Sunway; Selangor; Malaysia; Email: ong.chin.eng@monash.edu 
mediated drug metabolism for prediction of pharmacokinetic variability and the extent of drugdrug interactions. Examples of successful application of the in vitro and in silico approaches in delineating metabolism and interaction mechanism are presented. These approaches have generated valuable information and have proven their pivotal role in drug discovery, development and clinical use.

\section{In vitro APPROACHES}

The in vitro characterization of CYP-mediated activities can be conducted using simple and robust bioanalytical techniques, including the highthroughput methods (fluorescence and luminescence-based), medium-throughput methods (high performance liquid chromatography [HPLC] based and liquid chromatography-mass spectrometry [LC-MS] based) and low-throughput methods (radiometric-based). These bioanalytical techniques, coupled with in vitro enzyme assays after validation, can be used to produce reliable data for the determination of the kinetic parameters of the tested CYPs [3]. The choice of the method depends on the availability of equipment and technical support in each lab setting, as well as the sample size. The biochemical plots generated using the data obtained from each method can be used to elucidate the enzyme kinetics in the relevant CYP-mediated metabolism, either showing Michaelis-Menten kinetic or atypical kinetic (e.g., substrate activation and substrate inhibition). Kinetic models for DDIs can be derived based on the observed change in apparent constant $\left(\mathrm{K}_{\mathrm{m}}\right)$ which is determined from non-linear regression and statistical calculation. Inhibition kinetics such as $\mathrm{IC}_{50}$ (the inhibitor concentration causing $50 \%$ reduction on the enzyme activity) and $\mathrm{K}_{\mathrm{i}}$ (inhibition constant) can also be determined using the data generated from the in vitro CYP inhibition assay. The mode of inhibition of the enzyme (competitive, non-competitive, uncompetitive or mixed inhibition) is elucidated from Lineweaver-Burk plot, which is generated by plotting the reciprocal of velocity against the reciprocal of substrate concentration at different inhibitor concentrations $[4,5] . K_{i}$ value can also be determined from the secondary plot constructed by plotting the slopes (of each inhibitor concentration) from Lineweaver-Burk plot against the substrate [4]. Nonlinear regression analysis is nowadays the more commonly used way to estimating kinetics and inhibition parameters. Many commercially available software packages can be used for this purpose such as GraphPad Prism ${ }^{\circledR}$, SigmaPlot ${ }^{\circledR}$ (with Enzyme Kinetics module) and $\mathrm{GraFit}^{\circledR}$. These softwares provide valid regression algorithm and give robust parameter estimates. The intrinsic clearance of a drug (CL) can be calculated from $\mathrm{V}_{\max } / \mathrm{K}_{\mathrm{m}}$ value if the enzyme displays typical Michaelis-Menten kinetic. The detection of mechanism-based inhibition (MBI) is also made possible with in vitro CYP inhibition assays using CYP enzymes preincubated with and without the inhibitors [6]. Quantitative descriptors of MBI reaction include $\mathrm{K}_{\mathrm{I}}$ and $k_{\text {inact }}$ which are commonly used kinetic parameters describing the inactivation process. $\mathrm{K}_{\mathrm{I}}$ is the concentration of inactivator causing $50 \%$ of the maximal inactivation; whereas $\mathrm{k}_{\text {inact }}$ is the rate constant for maximal inactivation. To obtain these two parameters, the inactivation plot where time course of decline in enzyme activity at various inhibitor concentrations [I] is first plotted and fitted to a series of first-order decay curves. Estimates of the rate constant for enzyme inactivation $\left(\mathrm{k}_{\mathrm{obs}}\right)$ at the different inactivator concentrations can then be determined. $\mathrm{K}_{\mathrm{I}}$ and $\mathrm{k}_{\text {inact }}$ can subsequently be derived from the plot of $1 / k_{\text {obs }}$ against $1 /[\mathrm{I}]$.

A number of studies have established successful theoretical predictions of metabolic DDI in vivo by extrapolating the quantitative in vitro data [3, 7-10]. In order to predict the magnitude of DDIs, the ratio of area under the plasma concentration-time curve (AUC) of a victim drug are determined by using the in vivo inhibitor concentration and the in vitro inhibitor constant $\left([\mathrm{I}] / \mathrm{K}_{\mathrm{i}}\right.$ ratio). The AUC ratio $>2$ indicates a potential for DDI in vivo [11]. Utility of $[\mathrm{I}] / \mathrm{K}_{\mathrm{i}}$ approach to predict AUC ratio changes have had good success in predicting DDIs and over the years, its prediction accuracy has improved by careful selection of $[\mathrm{I}]$ and $\mathrm{K}_{\mathrm{i}}$ values of the drugs involved [12]. A representative listing of reports for prediction of DDIs from in vitro data is in Table 1. As shown in the table, the predictive methods have adequately predicted the extent of pharmacokinetic alteration and DDI risk and provided quantitative estimation of the effect of CYP-mediated drug interactions on drug substrate exposure. 
Table 1. Selected examples of in vitro studies for investigating CYP-mediated drug interaction or inhibition.

CYP isoform(s)

CYP3A4
Major findings of the study

Reference
This study attempted to use in vitro metabolism data to predict the effect of drugs with CYP3A4 inhibitory potency on tacrolimus exposure. The $\mathrm{IC}_{50}$ values of the investigated drugs on the formation of M-I, tacrolimus major metabolite, were calculated, and the effect on oral exposure $\left(\mathrm{AUC}_{\text {p.o. }}\right)$ of tacrolimus was assessed from static models (which examined changes in the AUC ratio by taking into consideration published pharmacokinetic parameters such as intestinal wall availability $\left[\mathrm{F}_{\mathrm{g}}\right]$, plasma unbound fraction $\left[\mathrm{F}_{\mathrm{a}}\right]$, and fraction of tacrolimus metabolized by CYP3A4 [ $\left.\left.\mathrm{f}_{\mathrm{m}(\mathrm{CYP} 3 \mathrm{~A} 4)}\right]\right)$. Most of the predicted increase in $\mathrm{AUC}_{\text {p.o. }}$. ratio fell within a 2-fold range of the observed values, indicating successful utilization of the models to predict DDI risk in patients using tacrolimus. Of all the CYP3A4 inhibitors examined, relatively high risk of interaction was correctly predicted for telaprevir, diltiazem and itraconazole.

Multiple CYP isoforms The in vitro DDI potential of SIPI5357, an arylalkanol-piperazine derivative used as an antidepressant, was investigated in this study. Potent inhibition of CYP2D6, CYP3A4 and CYP2C8 with low $\mathrm{IC}_{50}$ values (below $100 \mu \mathrm{M}$ ) but negligible inhibition of CYP1A2, CYP2C9, CYP2C19, CYP2E1 or CYP3A4 $\left(\mathrm{IC}_{50}>100 \mu \mathrm{M}\right)$ were observed for SIPI5357 in human liver microsomes. The compound showed a mixed mode inhibition of CYP2D6, the isoform with the highest inhibition susceptibility, with a $\mathrm{K}_{\mathrm{i}}$ of $11.12 \mu \mathrm{M}$. The value of [I]/K $\mathrm{K}_{\mathrm{i}}$ for SIPI5357 inhibition of CYP2D6 is below the US Food and Drug Administration (FDA) cut-off value of 0.1. Data from this study have indicated that SIPI5357 will not cause significant inhibition of CYP2D6 as well as other major CYP isoforms.

Multiple CYP isoforms In this study, seven dominant human liver CYP isoforms (CYP1A2, CYP2A6, CYP2B6, CYP2C9, CYP2C19, CYP2D6 and CYP3A4) were evaluated for inhibitory potential by antimalarial artemisinin and its structural derivatives, artesunate, artemether and dihydroartemisinin using recombinant enzymes and human liver microsomes. Nonlinear regression analysis was performed to delineate the inhibitory potency $\left(\mathrm{IC}_{50}\right.$ and $\left.\mathrm{K}_{\mathrm{i}}\right)$ and mechanisms of inhibition of the compounds. The risk of DDI in vivo was estimated from extrapolation using the $[\mathrm{I}] / \mathrm{K}_{\mathrm{i}}$ ratio. Inhibition was noted for all CYPs by all compounds tested, mostly through a mixed type of inhibition, with CYP1A2, CYP2B6, CYP2C19 and CYP3A4 being affected most. Substrates of CYP1A2 or CYP2C19 were predicted to demonstrate a high risk of interaction in vivo if artemisinin is coadministrated where AUC ratios $>4$ were noted when the maximum unbound concentrations of artemisinin at the inlet to the liver ([I $\left.]_{\max , \text { inlet, } \mathrm{u}}\right)$ were used in the extrapolation.

Multiple CYP isoforms This study focused on CYP-based DDI involving diethylstilbestrol (DES), a synthetic estrogen used for treating certain cases of prostatic and breast cancers. Given that DES might be co-administered with anticancer drugs, DES was examined for its CYP inhibitory effect in vitro, and the results demonstrated that DES was able to competitively inhibit CYP3A4, CYP2C8, CYP2C9 and CYP2E1 with $\mathrm{K}_{\mathrm{i}}$ values in single digit micromolar concentrations. Based on peak serum DES concentration after drip infusion of $500 \mathrm{mg}$ of fosfestrol (DES diphosphate) in patients, $[\mathrm{I}] / \mathrm{K}_{\mathrm{i}}$ ratios were calculated to be more than 2 for the four CYP isoforms, indicating potential of DES to induce in vivo DDI through CYP inhibition. 
Despite reasonable successes achieved using in vitro data, there are several confounding factors due to extrapolation from in vitro parameters which may contribute to inaccurate prediction. In a recent study by Sane and co-workers [17] which investigated the DDI potential of CYP3A4 probe substrate midazolam and the perpetrator drug deleobuvir, it was demonstrated that DDI prediction using deleobuvir alone had significantly over-predicted the DDI potential for CYP3A4 inhibition, with an AUC ratio of 6.15 , a ratio way higher than the clinically observed ratio of 1.23. Including metabolites data for two major metabolites, CD 6168 and deleobuvir-acyl glucuronide, into the predictive model brought the predicted inhibitory effect (AUC ratio of 0.97) closer to the observed value. This study shows that metabolites can significantly contribute to the DDI liability of the parent perpetrator compound and this may add uncertainty and complexity to the in vitro methods. In another recent study which examined the inhibition potential of faldaprevir, a selective inhibitor of hepatitis $\mathrm{C}$ virus NS3/4A protease, with major CYP isoforms [18], the DDI risk prediction based on $[\mathrm{I}] / \mathrm{K}_{\mathrm{i}}$ ratios was found to overestimate clinical effects while a net-effect model, which integrated other drug disposition and DDI mechanisms such as protein binding, gut and hepatic inhibition, provided more accurate prediction. The net-effect model predicted no interactions with faldaprevir for CYP2B6 and CYP2C9, and moderate inhibition for CYP3A4 (AUC ratios of 1.01, 1.02 and 3.06 respectively), in consistent with the clinical studies which demonstrated AUC ratio changes of 1.16, 1.29 and 2.92 for the three CYP isoforms respectively. On the other hand, the clinical effects were clearly overestimated using $[\mathrm{I}] / \mathrm{K}_{\mathrm{i}}$ values $(2.41$, 3.68 and 3.95 respectively). This indicates that factors such as protein binding which affects 'enzyme-available' inhibitor concentrations, as well as inhibition across multiple organs, could potentially complicate the predictive power of the in vitro models, and models that take the relevant confounding factors into account should provide better prediction for in vitro-in vivo scaling [19].

\section{In silico APPROACHES}

Using the state-of-the-art computational methods, the binding orientation and interactions of a specific ligand in CYP active site can be predicted virtually, followed by quantitative structure-activity relationship (QSAR) analysis. The increasing number of well-resolved crystal structures of human
CYP have contributed to the advancement of in silico molecular modeling and ligand docking in recent years [2, 20-23]. Ligand docking are made possible using various docking algorithms with different strengths and scoring functions, depending on the way the ligand is treated [24]. Validation of the molecular docking procedures is important to support reliable docking model for virtual screening [25]. Based on the postulated binding orientations and conformations, the possible interactions of the ligand with the active site residues of the enzyme can be elucidated, which provide insight into the binding specificity and mechanism of the enzyme towards the ligand [26]. Moreover, the docking models can provide quantitative information on the ranking of inhibition potencies of the inhibitors using the calculated interaction energy from each docked pose, which may complement the experimental data. QSAR analysis, on the other hand, attempts to relate chemical structures to the observed activities in a quantitative manner. 'Descriptors' are terms for the various calculated or measured properties and can be of many types (e.g., spatial, electronic, thermodynamic, conformational, topological and structural). These descriptors are 'linked-up' to generate equations or formulae to delineate structure-function activities in the CYP(s) under investigation [27-29].

Another computational approach involves the use of pharmacophore models that overlay ligand structures or their properties in 3D space in an attempt to elucidate the spatial, physical and chemical properties of CYP active or binding site. The models obtained are consensus structures that describe the electrostatic properties and size of the active sites, which are amenable for investigating detailed CYP-ligand interaction as well as structural modification of ligands to alter CYP binding [30]. Molecular dynamics (MD) simulation has emerged as an in silico technique that is increasingly used in CYP structure function analysis [31]. An important feature of MD is that it is able to simulate the flexible active site of CYPs in the order of nanoseconds time scale, thus accounting for the flexibility of this tool. The technique allows exploration of thermal motion, binding conformations and binding affinities of the ligands, as well as the motion, induced fit and conformational changes of CYP substrate access channel and active pocket [31-33]. With MD combined, docking result can be further strengthened. 
Table 2 lists recent studies using the abovementioned tools in examining CYP-ligand interaction. As is apparent from the table, different in silico methods have provided valuable insights into the structural and molecular basis of such interactions, including the dynamic aspects of protein structure, structural flexibility and plasticity of the ligand binding and catalysis, binding affinity determination, as well as structure-function relationship of the interactions. With further advancement of the techniques and increase in computational power, it is envisaged that in silico methods, serving to complement experimental investigation of drug metabolism, can and will continue to provide invaluable information to deepen understanding of CYP-ligand interactions.

\section{PERSPECTIVE AND SUMMARY}

The currently available in vitro and in silico methods for investigating CYP-mediated metabolism and interaction represent important experimental approaches in drug research. In vitro and in silico data provide important input to guide the design of clinical drug interaction investigations, prediction of trial outcomes, and to influence dose adjustment in drug labels and clinical practice. The obvious advantages offered by these tools include lower human risk due to the rational design of clinical studies, and in the case of new chemical entities, compound attrition during development can be reduced, with associated cost and time savings. Cost and time savings are particularly clear and alluring. With the rapid advancement of the in vitro screening systems and computer power and technologies, thousands of new chemical entitles can be screened and tested in a short period of time.

Table 2. Selected examples of in silico approaches for investigating ligand interactions of CYP enzymes.

\begin{tabular}{|c|c|c|c|}
\hline In silico approach & CYP isoform(s) & Major findings of the study & Reference \\
\hline \multirow[t]{2}{*}{$\begin{array}{l}\text { Protein-ligand } \\
\text { docking }\end{array}$} & $\begin{array}{l}\text { CYP2A6 and } \\
\text { CYP2A13 }\end{array}$ & $\begin{array}{l}\text { A structure-function study of naphthalene, phenanthrene, } \\
\text { biphenyl, and their analogues in interaction with CYP2A13 and } \\
\text { CYP2A6 using ligand binding spectroscopy and molecular } \\
\text { docking was undertaken. Spectral binding studies indicated that } \\
\text { CYP2A13 induced type I binding spectra more readily with the } \\
\text { examined chemicals when compared to CYP2A6. Molecular } \\
\text { docking demonstrated correlations between ligand interaction } \\
\text { energies (U values) for the chemicals with CYP2A13 and } \\
\text { CYP2A6 and the spectral binding efficiencies }\left(\Delta \mathrm{A}_{\mathrm{max}} / \mathrm{K}_{\mathrm{s}} \text { ratios). }\right. \\
\text { Those compounds having higher spectral binding efficiencies } \\
\text { (e.g., 2-ethynylnaphthalene, 3-ethynylphenanthrene and 2- } \\
\text { naphthalene propargyl ether) showed lower } \mathrm{U} \text { values, whereas } \\
\text { compounds which showed lower spectral binding efficiencies } \\
\text { had higher } \mathrm{U} \text { values. Data from this study support the } \\
\text { applicability of molecular docking analysis in investigating the } \\
\text { molecular interaction of xenobiotics with active sites of CYP } \\
\text { proteins. }\end{array}$ & [34] \\
\hline & CYP3A4 & $\begin{array}{l}\text { This study examined the effect of methamphetamine on spectral } \\
\text { binding, ligand docking and metabolism of protease inhibitors } \\
\text { (PIs) with CYP3A4. The data suggested that methamphetamine } \\
\text { did not alter the spectral change }\left(\Delta \mathrm{A}_{\max } \text { and } \mathrm{K}_{\mathrm{s}}\right) \text {, CYP3A4 } \\
\text { inhibition, and substrate docking of type I PIs (PIs that showed } \\
\text { type I spectral change in ligand binding spectral assay) with } \\
\text { CYP3A4. Molecular docking showed that there was no } \\
\text { significant difference in the average interaction energies of the } \\
\text { type I ligands in the presence and absence of methamphetamine. } \\
\text { Moreover, there was no major conformational change in the } \\
\text { active site of CYP3A4 with ligand binding in the presence of } \\
\text { methamphetamine. On the other hand, methamphetamine altered } \\
\text { spectral binding of type II PIs, ritonavir and indinavir, whereby } \\
\Delta \mathrm{A}_{\text {max }} \text { was decreased for ritonavir and } \mathrm{K}_{\mathrm{s}} \text { were reduced for both } \\
\text { PIs. Furthermore, in vitro incubations using human liver }\end{array}$ & [35] \\
\hline
\end{tabular}


Table 2. Continued...

microsomes showed a decrease in ritonavir hydroxylation in the presence of methamphetamine. Subsequent CYP3A4 docking with these PIs showed that methamphetamine altered the ligand docking in consistent with the data of the spectral binding and metabolism studies.

\begin{tabular}{|c|c|c|}
\hline \multirow[t]{2}{*}{ QSAR } & CYP1A2 & $\begin{array}{l}\text { A QSAR study was performed for } 21 \text { naturally occurring } \\
\text { flavonoids using both 2D (topological, structural and } \\
\text { thermodynamic) and 3D (spatial) descriptors to delineate } \\
\text { structural basis for potent CYP1A2 inhibition. The generated } \\
\text { QSAR models were of statistical significance both internally as } \\
\text { well as externally. The derived QSAR equations suggest that } \\
\text { double bond present at 2, 3-positions and the absence of hydroxyl } \\
\text { substituent or glycosidic linkage at 3-position of the 1,4- } \\
\text { benzopyrone nucleus were critical for CYP1A2 inhibition. } \\
\text { Furthermore, the phenyl ring present at 2-position of the 1,4- } \\
\text { benzopyrone ring should not be substituted with hydroxyl group. } \\
\text { Additionally, hydroxyl groups present in the benzopyran nucleus } \\
\text { at } 5 \text { and 7-positions should not be glycosylated for good CYP1A2 } \\
\text { inhibitory activity. }\end{array}$ \\
\hline & $\begin{array}{l}\text { CYP2C9 and } \\
\text { CYP2D6 }\end{array}$ & $\begin{array}{l}\text { This study used human clinical data as a base to construct four } \\
\text { QSAR models to recognize and identify substrates and inhibitors } \\
\text { for CYP2C9 and CYP2D6. The models were used to screen a } \\
\text { large data set of environmental chemicals and were able to } \\
\text { identify and discriminate substrates and inhibitors of the two } \\
\text { CYPs with high degree of concordance, specificity and } \\
\text { sensitivity ( } 66 \% \text { - } 88 \%) \text {. Compounds containing acidic functions } \\
\text { and aromatic amines were generally predicted to be CYP2C9 and } \\
\text { CYP2D6 inhibitors respectively. This supported the literature } \\
\text { findings in general. Furthermore, a weak overrepresentation of } \\
\text { polyaromatic hydrocarbons was seen among predicted CYP2C9 } \\
\text { active and Ames positive compounds, and the mutagenicity was } \\
\text { predicted with a QSAR model based on Ames in vitro test data. }\end{array}$ \\
\hline \multirow[t]{2}{*}{$\begin{array}{l}\text { Pharmacophore } \\
\text { modeling }\end{array}$} & CYP2C19 & $\begin{array}{l}\text { This study aimed to synthesize and investigate a series of } \\
\text { omeprazole-based analogues for inhibitory potency toward } \\
\text { CYP2C19. A CYP2C19 inhibition pharmacophore model was } \\
\text { built for the series, and additional analogues with inhibitory } \\
\text { potency against CYP2C19 were designed based on the model. } \\
\text { Upon identifying inhibitors for CYP2C19, ligand-based design } \\
\text { was adopted to develop compounds with improved metabolic } \\
\text { stability. A compound (compound 30) was identified to be a } \\
\text { highly potent and metabolically stable inhibitor for the isoform. }\end{array}$ \\
\hline & CYP3A4 & $\begin{array}{l}\text { A pharmacophore model for CYP3A4 specific inhibitor was built } \\
\text { based on the structure of ritonavir, a known CYP3A4 } \\
\text { pharmacoenhancer and inactivator. This study demonstrated that } \\
\text { the H-bond donor/acceptor moiety, a flexible backbone, and } \\
\text { aromaticity of the side group analogous to Phe-2 of ritonavir } \\
\text { were important for CYP3A4 inhibition. Hydrophobic } \\
\text { interactions at the sites adjacent to the haem and phenylalanine } \\
\text { cluster were also pivotal in the ligand binding process. } \\
\text { Furthermore, two compounds that were less complex than } \\
\text { ritonavir but showed comparable inhibitory potency were } \\
\text { identified. }\end{array}$ \\
\hline $\begin{array}{l}\text { Molecular } \\
\text { dynamics }\end{array}$ & CYP1A2 & $\begin{array}{l}\text { The inhibitory mechanism against CYP1A2 for oroxylin and } \\
\text { wogonin, two biologically active flavonoids found in commonly }\end{array}$ \\
\hline
\end{tabular}


Table 2. Continued...

used traditional Chinese herbal medicine, were investigated using molecular dynamics simulations together with molecular docking. The in silico simulations revealed that van der Waals, hydrophobic and hydrogen-bonding interactions were important in the inhibitory mechanisms of oroxylin. Oroxylin binding also induced a larger and more open binding site in CYP1A2-oroxylin complex compared to that of wogonin and $\alpha$-naphthoflavone, a potent CYP1A2 inhibitor probe. Unlike oroxylin, binding of wogonin and $\alpha$-naphthoflavone involved mainly hydrogenbonding as the predominant force. The different binding pocket architecture and binding mode have accounted for the relatively weaker inhibitory effect of oroxylin.

CYP3A4 This study used molecular dynamics simulations, together with [41] free-energy calculations, to investigate the positive homotropic cooperativity of ketoconazole binding in CYP3A4. The MD simulations demonstrated that the binding of the first ketoconazole molecule was able to increase the binding affinity for the second molecule. The major driving force for this binding was the shape complementarity through nonpolar van der Waals interactions. Furthermore, the calculated binding free energies were found to agree well with the values predicted from a simple 2-ligand binding kinetic model. Also, the calculated values were broadly similar to the experimentally derived data.

As a consequence of high data throughput, the modest instrumentation requirements, and more efficient actual clinical study protocols, the overall cost of developing and maintaining in vitro and in silico programs generally is low or modest.

Despite various advantages and benefits, the in vitro and in silico methods are not without their limitation and drawbacks. The drawback mainly stem from the complexities and confounding factors when extrapolating in vitro and in silico data to in vivo situations. A number of uncertainties and sources of bias and error in the in vitro methods have been demonstrated, including the inhibitor existing as enantiomers in vivo, the metabolites of the inhibitor also affecting the pharmacokinetic of the victim drug, uncertainties in estimating enzymeavailable inhibitor concentrations, cooperativity and allosteric regulation of CYPs, non-CYP determinants of metabolic clearance, and concurrent induction and inhibition [12]. For the in silico approaches, certain confounding factors may complicate the task of developing appropriate models. As an example, CYP3A4, known for its large binding site and ability to accommodate different binding modes of diverse chemical structures, necessitates complex modeling efforts and requires techniques to deconvolute the resulting mixed data content. Moreover, not only can small ligands interact as substrates or inhibitors, heteroactivation through a unique binding location within the CYP3A4 pocket can further complicate the modeling task [27]. In addition, the generally lack of complete information of stereochemical and conformational data of CYP protein and the structural characteristics of ligand, in particular, the structural information of the active sites of the more recently identified CYPs, have made the application of in silico methods restricted somewhat [27]. In a recent study whereby QSAR modeling of major CYP isoforms was utilized to predict likelihood of DDI for all possible binary combinations of marketed drugs [42], Zakharov and co-workers were able to demonstrate good accuracy of prediction (balanced accuracy exceeding $70 \%$ for more than 600,000 combinations) using their developed models but felt that the accuracy of the models could be improved further if additional factors underlying DDI mechanisms such as concurrent inhibition and induction, non-CYP clearance and transport mechanisms and drug intrinsic pharmacokinetic properties could be integrated into the computational models. Lonsdale and his colleagues recently used 
quantum mechanics/molecular mechanics (QM/MM) methods to examine mechanism of Nhydroxylation of $(R)$-mexiletine by CYP1A2. They demonstrated that the calculated reaction barriers derived from $\mathrm{QM} / \mathrm{MM}$ pointed to direct $\mathrm{N}$-oxidation as the preferred mechanism over that of $\mathrm{H}$ abstraction/rebound. They further opined that the reliability of their results could be further enhanced by determining reaction barrier profiles for mechanism and spin state of more starting geometries of mexiletine during QM/MM modeling. This was however not practical due to high computational cost, indicating that running cost may restrain somewhat the extensive iterations needed for better predictive precision using in silico methods [43]. Taken together, the abovementioned complexities and confounding factors have affected to varying degree the applicability and predictive performance of current in vitro and in silico models.

The challenge of developing the best possible in vitro and in silico methodologies will remain as more is learned about CYP enzymes and, as more data on ligand interactions with the enzymes are reported, existing tools and methods need to be validated, refined and additional tools will be created. These tools have aided greatly to better understanding of the nature of CYP-mediated metabolism and interaction, and their further expansion will continue to contribute to the process of drug discovery, development and ultimately safer and more effective pharmacotherapy.

\section{ACKNOWLEDGEMENTS}

The planning and preparation of this review were supported by the FRGS Grant (no. FRGS/1/2014/SKK03/MUSM/02/1) of the Ministry of Higher Education, Malaysia.

\section{CONFLICT OF INTEREST}

All authors wish to declare that they have no conflict of interest or competing interest relevant to the content of this article.

\section{REFERENCES}

1. Schroer K, KittelmannI M, Lütz S. Recombinant human cytochrome P450 monooxygenases for drug metabolite synthesis. Biotechnol Bioeng. 2010; 106: 699-706. DOI: 10.1002/bit.22775.

2. Czodrowski P, Kriegl JM, Scheuerer S, Fox T. Computational approaches to predict drug metabolism. Ex Opin Drug Metab Toxicol. 2009; 5: 15-27. DOI: $10.1517 / 17425250802568009$.

3. Foti RS, Wienkers LC, Wahlstrom JL. Application of cytochrome P450 drug interaction screening in drug discovery. Comb Chem High T Scr. 2010; 13: 14558.

4. Tang J-C, Yang H, Song X-Y, Song X-H, Yan S-L, Shao J-Q, Zhang T-L, Zhang J-N. Inhibition of cytochrome $\mathrm{P} 450$ enzymes by rhein in rat liver microsomes. Phytother Res. 2009; 23: 159-64. DOI: $10.1002 /$ ptr.2572.

5. Kimura $\mathrm{Y}$, Ito $\mathrm{H}$, Ohnishi R, Hatano T. Inhibitory effects of polyphenols on human cytochrome P450 3A4 and 2C9 activity. Food Chem Toxicol. 2010; 48: 429-35. DOI: 10.1016/j.fct.2009.10.041.

6. Zhou S, Chan SY, Goh BC, Chan E, Duan W, Huang M, McLeod HL. Mechanism-based inhibition of cytochrome P450 3A4 by therapeutic drugs. Clin Pharmacokinet. 2005; 44: 279-304.

7. Yao C, Levy RH. Inhibition-based metabolic drugdrug interactions: predictions from in vitro data. $\mathrm{J}$ Pharm Sci. 2002; 91: 1923-35.

8. Wienkers LC, Heath TG. Predicting in vivo drug interactions from in vitro drug discovery data. Nat Rev Drug Discov. 2005; 4: 825-33.

9. McGinnity DF, Waters NJ, Tucker J, Riley RJ. Integrated in vitro analysis for the in vivo prediction of cytochrome P450-mediated drug-drug interactions. Drug Metab Dispos. 2008; 36: 1126-34. DOI: 10.1124/dmd.108.020446.

10. Sekiguchi N, Higashida A, Kato M, Nabuchi Y, Mitsui T, Takanashi K, Aso Y, Ishigai M. Prediction of drug-drug interactions based on time-dependent inhibition from high throughput screening of cytochrome P450 3A4 inhibition. Drug Metab Pharmacokinet. 2009; 24: 500-10.

11. Obach RS, Walsky RL, Venkatakrishnan K, Gaman EA, Houston JB, Tremaine LM. The utility of in vitro cytochrome $\mathrm{P} 450$ inhibition data in the prediction of drug-drug interactions. J Pharmacol Exp Ther. 2006; 316: 336-48.

12. Obach RS. Predicting clearance in humans from in vitro data. Curr Top Med Chem. 2011; 11: 334-9.

13. Oda $\mathrm{K}$, Yamano $\mathrm{K}$, Otsuka $\mathrm{Y}$. In vitro-in vivo extrapolations to evaluate the effect of concomitant drugs on tacrolimus (FK506) exposure. Biopharm Drug Dispos. 2015; 35: 265-74. DOI: 10.1002/bdd.1938.

14. Fan G, Cao Y, Yang J. In vitro inhibition and induction of human cytochrome P450 enzymes by SIPI5357, a potential antidepressant. Biopharm Drug Dispos. 2015; 36: 364-72. DOI: 10.1002/bdd.1947.

15. Ericsson T, Sundell J, Torkelsson A, Hoffmann KJ, Ashton M. Effects of artemisinin antimalarials on cytochrome P450 enzymes in vitro using recombinant enzymes and human liver microsomes: potential implications for combination therapies. Xenobiotica. 


2014; $\quad 44: \quad 615-26 . \quad$ DOI:
$10.3109 / 00498254.2013 .878815$.

16. Qu YQ, Fang ZZ, Yang L, Gao ZM, Liang R, Zhu LL, Dong PP, Zhang YY, Ge GB, Wang LM. Reversible inhibition of four important human liver cytochrome P450 enzymes by diethylstilbestrol. Pharmazie. 2011; 66: 216-21.

17. Sane RS, Ramsden D, Sabo JP, Cooper C, Rowland L, Ting N, Whitcher-Johnstone A, Tweedie DJ. Contribution of major metabolites toward complex drug-drug interactions of deleobuvir: In vitro predictions and in vivo outcomes. Drug Metab Dispos. 2016; 44: 466-75. DOI: 10.1124/dmd.115.066985.

18. Sabo JP, Kort J, Ballow C, Kashuba AD, Haschke M, Battegay M, Girlich B, Ting N, Lang B, Zhang W, Cooper C, O'Brien D, Seibert E, Chan TS, Tweedie $\mathrm{D}, \mathrm{Li}$ Y. Interactions of the hepatitis $\mathrm{C}$ virus protease inhibitor faldaprevir with cytochrome P450 enzymes: in vitro and in vivo correlation. J Clin Pharmacol. 2015; 55: 467-77. DOI: 10.1002/jcph.436.

19. Vieira ML, Kirby B, Ragueneau-Majlessi I, Galetin A, Chien JY, Einolf HJ, Fahmi OA, Fischer V, Fretland A, Grime K, Hall SD, Higgs R, Plowchalk D, Riley R, Seibert E, Skordos K, Snoeys J, Venkatakrishnan K, Waterhouse T, Obach RS, Berglund EG, Zhang L, Zhao P, Reynolds KS, Huang SM. Evaluation of various static in vitro-in vivo extrapolation models for risk assessment of the CYP3A inhibition potential of an investigational drug. Clin Pharmacol Ther 2014; 95: 189-98. DOI: 10.1038/clpt.2013.187.

20. Dai Y, Wang Q, Zhang X, Jia S, Zheng H, Feng D, $\mathrm{Yu}$ P. Molecular docking and QSAR study on steroidal compounds as aromatase inhibitors. Eur J Med Chem. 2010; 45: 5612-20. DOI: 10.1016/j.ejmech.2010.09.011.

21. Cojocaru V, Balali-Mood K, Sansom MS, Wade RC. Structure and dynamics of the membrane-bound cytochrome P450 2C9. PLoS Comp Biol. 2011; 7: e1002152. DOI: 10.1371/journal.pcbi.

22. Denisov I, Shih A, Sligar S. Structural differences between soluble and membrane bound cytochrome P450s. J Inorg Biochem. 2012; 108: 150-8. DOI: 10.1016/j.jinorgbio.2011.11.026.

23. Mo S-L, Liu W-F, Chen Y, Luo H-B, Sun L-B, Chen X-W, Zhou Z-W, B Sneed K, Guang Li C, Du Y-M. Ligand-and protein-based modeling studies of the inhibitors of human cytochrome P450 2D6 and a virtual screening for potential inhibitors from the Chinese herbal medicine, Scutellaria baicalensis (Huangqin, Baikal Skullcap). Comb Chem High T Scr. 2012; 15: 36-80.

24. Kroemer RT. Structure-based drug design: docking and scoring. Curr Protein Pept Sci. 2007; 8: 312-28.

25. Hevener KE, Zhao W, Bali DM, Babaoglu K, Qi J, White SW, Lee RE. Validation of molecular docking programs for virtual screening against dihydropteroate synthase. J Chem Inf Model. 2009; 49: 444-60. DOI: 10.1021/ci800293n.

26. Dhers L, Pietrancosta N, Ducassou L, Ramassamy B, Dairou J, Jaouen M, André F, Mansuy D, Boucher JL. Spectral and 3D model studies of the interaction of orphan human cytochrome P450 2U1 with substrates and ligands. Biochim Biophys Acta. 2017; 1861(1 Pt A): 3144-53. DOI: 10.1016/j.bbagen.2016.07.018.

27. Zhang T, Chen Q, Li L, Angela Liu L, Wei D-Q. In silico prediction of cytochrome $\mathrm{P} 450$-mediated drug metabolism. Comb Chem High T Scr. 2011; 14: 38895.

28. Raunio H, Juvonen RO, Poso A, Lahtela-Kakkonen M, Rahnasto-Rilla M. Common and distinct interactions of chemical inhibitors with cytochrome P450 CYP1A2, CYP2A6 and CYP2B6 enzymes. Drug Metab Lett. 2016; 10: 56-64.

29. Nembri S, Grisoni F, Consonni V, Todeschini R. In Silico prediction of cytochrome P450-drug interaction: QSARs for CYP3A4 and CYP2C9. Int J Mol Sci. 2016; 17: 914. DOI: 10.3390/ijms17060914.

30. Vermeulen NPE. Alternate prediction of drug metabolism: the case of cytochrome P450 2D6. Curr Top Med Chem. 2003; 3: 1227-39.

31. Nair PC, McKinnon RA, Miners JO. Cytochrome P450 structure-function: insights from molecular dynamics simulations. Drug Metab Rev. 2016; 48: 434-52. DOI: 10.1080/03602532.2016.1178771.

32. Dodani SC, Kiss G, Cahn JKB, Su Y, Pande VS, Arnold FH. Discovery of a regioselectivity switch in nitrating $\mathrm{P} 450 \mathrm{~s}$ guided by molecular dynamics simulations and Markov models. Nat Chem. 2016; 8: 419-25. DOI: 10.1038/NCHEM.2474.

33. Kesharwani SS, Nandekar PP, Pragyan P, Rathod V, Sangamwar AT. Characterization of differences in substrate specificity among CYP1A1, CYP1A2 and CYP1B1: an integrated approach employing molecular docking and molecular dynamics simulations. J Mol Recognit. 2016; 29: 370-90. DOI: 10.1002/jmr.2537.

34. Shimada T, Takenaka S, Kakimoto K, Murayama N, Lim YR, Kim D, Foroozesh MK, Yamazaki H, Guengerich FP, Komori M. Structure-function studies of naphthalene, phenanthrene, biphenyl, and their derivatives in interaction with and oxidation by cytochromes P450 2A13 and 2A6. Chem Res Toxicol. 2016; 29: 1029-40. DOI: 10.1021/acs.chemrestox.6b00083.

35. Nookala AR, Li J, Ande A, Wang L, Vaidya NK, Li W, Kumar S, Kumar A. Effect of methamphetamine on spectral binding, ligand docking and metabolism of anti-HIV drugs with CYP3A4. PLoS ONE 2016; 11: e0146529. DOI: 10.1371/journal.pone.0146529

36. Roy K, Roy PP. 2008. Comparative QSAR studies of CYP1A2 inhibitor flavonoids using 2D and 3D 
descriptors. Chem Biol Drug Des. 2008; 72: 370-82. DOI: $10.1111 / \mathrm{j} .1747-0285.2008 .00717 . x$.

37. Jónsdóttir SÓ, Ringsted T, Nikolov NG, Dybdahl M, Wedebye EB, Niemelä JR. Identification of cytochrome P450 2D6 and 2C9 substrates and inhibitors by QSAR analysis. Bioorg Med Chem. 2012; 20: 2042-53. DOI: 10.1016/j.bmc.2012.01.049.

38. Foti RS, Rock DA, Han X, Flowers RA, Wienkers LC, Wahlstrom JL. Ligand-based design of a potent and selective inhibitor of cytochrome P450 2C19. J Med Chem. 2012; 55: 1205-14. DOI: $10.1021 / \mathrm{jm} 201346 \mathrm{~g}$.

39. Kaur P, Chamberlin AR, Poulos TL, Sevrioukova IF. Structure-based inhibitor design for evaluation of a CYP3A4 pharmacophore model. J Med Chem. 2016; 59: 4210-20. DOI: 10.1021/acs.jmedchem.5b01146.

40. Shao YX, Zhao P, Li Z, Liu M, Liu P, Huang M, Luo HB. The molecular basis for the inhibition of human cytochrome P450 1A2 by oroxylin and wogonin. Eur Biophys J. 2012; 41: 297-306. DOI: 10.1007/s00249011-0785-1.

41. Bren U, Oostenbrink C. Cytochrome P450 3A4 inhibition by ketoconazole: tackling the problem of ligand cooperativity using molecular dynamics simulations and free-energy calculations. J Chem Inf Model. 2012; 52: 1573-82. DOI: 10.1021/ci300118x.

42. Zakharov AV, Varlamova EV, Lagunin AA, Dmitriev AV, Muratov EN, Fourches D, Kuz'min VE, Poroikov VV, Tropsha A, Nicklaus MC. QSAR modeling and prediction of drug-drug interactions. Mol Pharm. 2016; 13: 545-56. DOI: 10.1021/acs.molpharmaceut.5b00762.

43. Lonsdale R,Fort RM, Rydberg P, Harvey JN, Mulholland AJ. Quantum mechanics/molecular mechanics modeling of drug metabolism: mexiletine N-hydroxylation by cytochrome P450 1A2. Chem Res Toxicol. 2016; 29: 963-71. DOI: 10.1021/acs.chemrestox.5b00514. 